EPJ Web of Conferences 37, 01020 (2012)

DOI: $10.1051 /$ epjconf/20123701020

(C) Owned by the authors, published by EDP Sciences, 2012

\title{
Near-threshold pion production in diproton reactions with polarised beams and target at ANKE-COSY
}

\author{
S. Dymov ${ }^{1,2, a}$ for the ANKE Collaboration \\ 1 Physikalisches Institut II, Universität Erlangen-Nürnberg, 91058 Erlangen, Germany \\ 2 Laboratory of Nuclear Problems, Joint Institute for Nuclear Research, 141980 Dubna, Russia
}

\begin{abstract}
An extensive experimental study of near-threshold pion production in diproton reactions is underway at the ANKE-COSY spectrometer (Jülich). The programme is aimed at isolating the four-nucleon-pion contact interaction term appearing in the $\chi P T$ expansions of these processes. This will establish links between pion production and other low energy phenomena within the $\chi P T$ approach. The first step in the programme was to measure the differential cross-section and the proton analysing power in the $\vec{p} p \rightarrow\{p p\}_{s} \pi^{0}$ and $\vec{p} n \rightarrow\{p p\}_{s} \pi^{-}$reactions over the full angular range. Here $\{p p\}_{s}$ denotes a diproton, i.e., a two-proton system in a ${ }^{1} S_{0}$ state. These data allow a partial wave analysis to be carried out provided that simplifying assumptions are made when applying the Watson theorem. To make the analysis more robust, and independent of the uncertainties in the relative normalization, the spin-correlation coefficients $A_{x, x}$ and $A_{y, y}$ in the $\vec{n} \vec{p} \rightarrow\{p p\}_{s} \pi^{-}$reaction were measured in a follow-up experiment. The first results of the data analysis are presented and future developments of the programme are outlined.
\end{abstract}

\section{The physics case for near-threshold single pion production}

The ANKE [1] experimental programme on the near-threshold pion production aims to measure the cross sections and spin observables in the $p p \rightarrow\{p p\}_{s} \pi^{0}$ and $n p \rightarrow\{p p\}_{s} \pi^{-}$reactions [2,3]. The symbol $\{p p\}_{s}$ here denotes a diproton, that is, an unbound proton pair with a very low excitation energy, $E_{p p}<3 \mathrm{MeV}$. The selection of a low excitation energy ensures the dominance of the ${ }^{1} S_{0}$ state of the diproton, which simplifies significantly the theoretical analysis. A full data set of all observables at low beam energies would allow us to determine the partial wave amplitudes which, in turn, would provide a non-trivial test of chiral perturbation theory [4] and also lead to the determination of the value of the parameter $d$, which represents the important contact term that affects the pion $p$-wave amplitudes.

The short range physics in chiral effective field theories, which provide a model-independent understanding of Nature, is encoded in the so-called low energy constants (LEC). These LECs, once determined from one process, can be applied to predict many others. For example, the LECs $c 1-c 4$ extracted from $\pi N$ analysis on the basis of chiral perturbation theory are now widely used to parameterise the short range physics in the $N N$-interaction, few-nucleon systems, single (and multi-) pion production in $N N$ collisions etc. Analogously, by studying the $p$-wave pion production amplitudes we get access to the $4 N \pi$ contact operator, the strength of which is controlled by the low energy constant $d$. This LEC enters also in electroweak processes, such as $p p \rightarrow d e^{+} v$ and triton $\beta$ decay, in few-body operators $\left(e . g\right.$. in $p d \rightarrow p d$ ), pion photoproduction $\gamma d \rightarrow n n \pi^{+}$and its inverse $\pi d \rightarrow \gamma N N$. It therefore plays a very important role in connecting different low-energy reactions.

On the practical side, the $p p \rightarrow\{p p\}_{s} \pi^{0}$ and $n p \rightarrow\{p p\}_{s} \pi^{-}$reactions have the big advantage for COSY that both the pion and diproton have spin-zero, which means that the only spin degrees of free-

a e-mail: s.dymov@fz-juelich.de unrestricted use, distribution, and reproduction in any medium, provided the original work is properly cited. 
dom are connected with the initial state. There are therefore no non-trivial spin-transfer observables, which means that rescattering experiments are not required.

Four types of experiments are possible for both $\pi^{0}$ and $\pi^{-}$production. These are the measurement of the unpolarised differential cross section $d \sigma / d \Omega$, the beam or target analysing power $A_{y}$, the in-plane spin-correlation $A_{x, x}$, and the mixed correlation parameter $A_{x, z}$. Knowing these one can determine the magnitudes and the relative phase of the two scalar amplitudes as functions of the pion production angle for either the $p p$ or $p n$ experiment.

At low energies it is reasonable to assume that data can be analysed by truncating the partial wave expansion at orbital angular momentum $\ell=2$. It is shown in [5] that the magnitude of one of the $p$ wave amplitudes is then fixed completely by the measurement of $\left(1-A_{x, x}\right) \cdot d \sigma / d \Omega$ for $n p \rightarrow\{p p\}_{s} \pi^{-}$ and that the magnitude of the other $p$-wave amplitude and its relative phase can be deduced from a combined analysis of this with our cross section and analysing power data for $p p \rightarrow\{p p\}_{s} \pi^{0}$ and $n p \rightarrow\{p p\}_{s} \pi^{-}$. These data will provide two determinations of the LEC $d$. Measurements of the mixed spin-correlation parameters $A_{z, x}$ are not required for the extraction of the $p$-wave amplitudes, though such information is vital in order to identify the $d$-wave terms.

\section{Measurement of cross section and analysing power}

As first steps in the programme, measurements with a polarised proton beam incident on unpolarised hydrogen and deuterium cluster targets were performed at a beam energy of $T_{p}=353 \mathrm{MeV}[2,3]$.

\subsection{The $\vec{p} p \rightarrow\{p p\}_{s} \pi^{0}$ process}

The polarised proton COSY beam and the ANKE hydrogen cluster target were used in this measurement. The final proton pair was recorded in the ANKE forward detector and identified with the use of the time-of-flight difference between the two protons. After the selection of the proton pairs, the $\vec{p} p \rightarrow\{p p\}_{s} \pi^{0}$ events were identified by the missing-mass criterion. The beam polarisation and luminosity were determined by detecting simultaneously the $\vec{p} p \rightarrow d \pi^{+}$and $\vec{p} p \rightarrow p p$ processes, for which the cross sections and analysing powers are known with high precision at this energy.

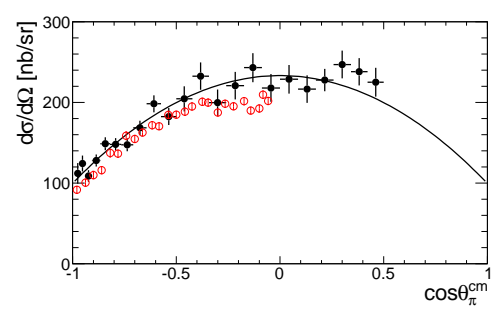

Fig. 1. Differential cross section for the $p p \rightarrow\{p p\}_{s} \pi^{0}$ reaction at $353 \mathrm{MeV}$ as a function of the cosine of the pion centre-of-mass angle. Solid (black) circles represent ANKE measurements [6]. Open (red) circles are CELSIUS data obtained at $360 \mathrm{MeV}$ [7]. The curve is a linear fit in $\cos ^{2} \theta_{\pi}$ to our data.

The ANKE results for the differential cross section and the vector analysing power are shown in Figs. 1 and 2. For a spin-singlet diproton, the spin structure of the $p p \rightarrow\{p p\}_{s} \pi^{0}$ or $n p \rightarrow\{p p\}_{s} \pi^{-}$reaction is that of $\frac{1}{2}^{+} \frac{1}{2}^{+} \rightarrow 0^{+} 0^{-}$. Parity and angular momentum conservation require the initial nucleonnucleon pair to have spin $S=1$. The pion orbital angular momentum $\ell$ and the initial nucleon-nucleon isospin $I$ are then linked by $\ell+I=$ odd so that, for the $p p \rightarrow\{p p\}_{s} \pi^{0}$ reaction, only even pion partial waves are allowed. As a consequence, the unpolarised cross section for $\pi^{0}$ production, and this times 

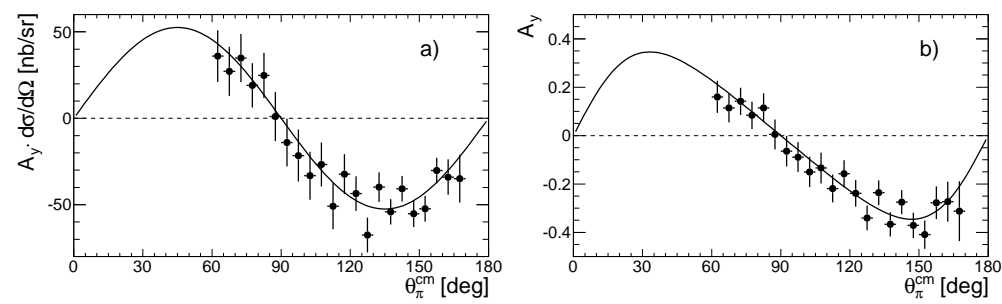

Fig. 2. (a) The product of the measured analysing power and differential cross section for the $\vec{p} p \rightarrow\{p p\}_{s} \pi^{0}$ reaction. The curve represents the best fit of Eq. (1), keeping terms up to $b_{2}$.

(b) Measured values of $A_{y}$ for the $\vec{p} p \rightarrow\{p p\}_{s} \pi^{0}$ reaction [6]. The line represents the quotient of the best fit to the data in panel (a) and the fit to the cross section of Fig. 1.

the proton analysing power $A_{y}$, must be of the form

$$
\begin{aligned}
\left(\frac{d \sigma}{d \Omega}\right) & =\frac{k}{4 p}\left(a_{0}+a_{2} \cos ^{2} \theta_{\pi}+a_{4} \cos ^{4} \theta_{\pi}+\cdots\right), \\
A_{y}\left(\frac{d \sigma}{d \Omega}\right)_{0} & =\frac{k}{4 p} \sin \theta_{\pi} \cos \theta_{\pi}\left(b_{2}+b_{4} \cos ^{2} \theta_{\pi}+\cdots\right),
\end{aligned}
$$

where $\theta_{\pi}$ is the pion c.m. production angle with respect to the direction of the polarised proton beam. Here $p$ is the incident c.m. momentum and $k$ that of the produced pion which, at $353 \mathrm{MeV}$, have values $p=407 \mathrm{MeV} / c$ and $k \approx 94 \mathrm{MeV} / c$. If one considers only pion waves with $l \leq 2$, a non-zero value of the analysing power in this process must arise from the interference between the $s$ and $d$ waves. The strong $A_{y}$ signal demonstrates the importance of this interference.

\subsection{The $\vec{p} n \rightarrow\{p p\}_{s} \pi^{-}$process}

The ANKE deuterium cluster target was used in the experiment. Low energies of the spectator proton, $T_{\text {spec }}<6 \mathrm{MeV}$, were selected to ensure the validity of the quasi-free approximation. The diproton was recorded in either the Forward or Positive side ANKE detectors. In addition, either the $\pi^{-}$was recorded at large c.m. angles in the Negative side Detector, or the spectator proton was measured in the Silicon Tracking Telescope (STT). Particle identification was made on the basis of the time-offlight information except for the spectator proton, which was recognised through its energy loss in the STT. The process was then isolated via the missing mass value built for the three observed final state particles. The polarisation of the proton beam and the reaction luminosity were both estimated from the quasi-free $\vec{p} n \rightarrow d \pi^{0}$ data that were taken in parallel.

The unpolarised cross section for $\pi^{-}$production, and this times the proton analysing power $A_{y}$, are of the form

$$
\left(\frac{d \sigma}{d \Omega}\right)_{0}=\frac{k}{4 p} \sum_{n=0} a_{n} \cos ^{n} \theta_{\pi}, \quad A_{y}\left(\frac{d \sigma}{d \Omega}\right)_{0}=\frac{k}{4 p} \sin \theta_{\pi} \sum_{n=0} b_{n+1} \cos ^{n} \theta_{\pi},
$$

where $\theta_{\pi}$ is the pion c.m. production angle, $p$ is the incident c.m. momentum, and $k$ that of the produced pion.

Whereas the TRIUMF results only cover the central region of pion angles [9], the ANKE data [8] in Fig. 3 extend over the whole angular domain. The two data sets are consistent in the backward hemisphere but the TRIUMF measurements show no indication of the rise at forward angles that is seen at ANKE. Some confirmation of the ANKE angular shape is offered by pion absorption data, $\pi^{-3} \mathrm{He} \rightarrow p n p_{\text {spec }}$, where the unobserved slow proton is assumed to be a spectator [10]. In this case the reaction can be interpreted as being $\pi^{-}\{p p\}_{s} \rightarrow p n$, though the internal structure of the bound diproton is very different to that in the production data. The forward/backward peaking is in complete contrast to the results found for $\pi^{0}$ production [7] and is an indication of the dominance of the $I=0 p$-wave amplitudes in this reaction. 


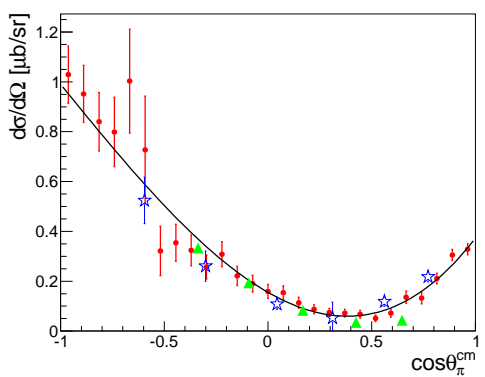

Fig. 3. Unpolarised differential cross section for the $p n \rightarrow\{p p\}_{s} \pi^{-}$reaction at $\approx 353 \mathrm{MeV}$. The ANKE data [8] are shown by red circles and the TRIUMF results [9] by green triangles. The arbitrarily scaled TRIUMF cross sections extracted from $\pi^{-3} \mathrm{He} \rightarrow p n p_{\text {spec }}$ data [10] are also included (blue stars). The curve is a cubic fit to the ANKE data.
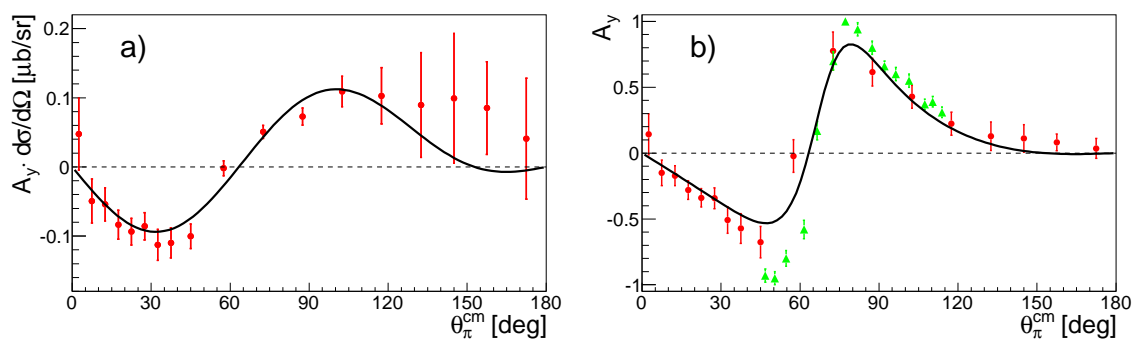

Fig. 4. (a) The product of the measured analysing power and differential cross section for the $\vec{p} n \rightarrow\{p p\}_{s} \pi^{-}$ reaction at $353 \mathrm{MeV}$. The curve represents the best fit of Eq. (2) with $b_{1}, b_{2}$, and $b_{3}$ terms. (b) Measured values of $A_{y}$ for the $\vec{p} n \rightarrow\{p p\}_{s} \pi^{-}$reaction showing both the ANKE (circles) [8] and TRIUMF data [11] (triangles). The line represent the quotient of the fit in panel (a) and that to the cross section in Fig. 3.

The results for the analysing power of the $\vec{p} n \rightarrow\{p p\}_{s} \pi^{-}$reaction [8] are displayed in Fig. 4, with $A_{y}(d \sigma / d \Omega)$ being shown in panel (a) and $A_{y}$ in panel (b). The agreement with the TRIUMF $A_{y}$ data [11] is good in the backward hemisphere and both show a strong and rather asymmetric fluctuation in the central angular region.

\subsection{Partial wave analysis}

The spin structure of the $p n \rightarrow\{p p\}_{s} \pi^{-}$and of $p p \rightarrow\{p p\}_{s} \pi^{0}$ reactions are identical. The cross section and analysing power can be written in terms of two scalar amplitudes $A$ and $B$;

$$
\left(\frac{d \sigma}{d \Omega}\right)_{0}=\frac{k}{4 p}\left(|A|^{2}+|B|^{2}+2 \operatorname{Re}\left[A B^{*}\right] \cos \theta_{\pi}\right), \quad A_{y}\left(\frac{d \sigma}{d \Omega}\right)_{b}=\frac{k}{4 p}\left(2 \operatorname{Im}\left[A B^{*}\right] \sin \theta_{\pi}\right) .
$$

Keeping terms up to pion $d$ waves, the $p p \rightarrow\{p p\}_{s} \pi^{0}$ data at $353 \mathrm{MeV}$ [6] can be parameterised in terms of the three partial wave amplitudes $M_{s}^{P}, M_{d}^{P}$, and $M_{d}^{F}$, corresponding to the transitions, ${ }^{3} P_{0} \rightarrow{ }^{1} S_{0} s,{ }^{3} P_{2} \rightarrow{ }^{1} S_{0} d$, and ${ }^{3} F_{2} \rightarrow{ }^{1} S_{0} d$, respectively. In proton-neutron collisions there are also the two $p$-wave transitions, ${ }^{3} S_{1} \rightarrow{ }^{1} S_{0} p$ and ${ }^{3} D_{1} \rightarrow{ }^{1} S_{0} p$ that arise in the isospin $I=0$ case, and for these we introduce amplitudes $M_{p}^{S}$ and $M_{p}^{D}$, respectively. It is clear from the very different behaviour of the angular distributions for $\pi^{0}$ and $\pi^{-}$production that the extra $p$-wave amplitudes are very large and that it will not be justified to discard their interference with $M_{d}^{P}$.

By neglecting the small coupling between the ${ }^{3} P_{2}$ and ${ }^{3} F_{2}$ partial waves, and imposing the Watson theorem to determine the phases, it was possible to extract values for the complex amplitudes $M_{s}^{P}, M_{d}^{P}$, and $M_{d}^{F}$ from the analysis of the $p p \rightarrow\{p p\}_{s} \pi^{0}$ reaction [6]. Such an approach is not valid for the two 
$p$-wave terms because of the very strong coupling between the incident ${ }^{3} S_{1}$ and ${ }^{3} D_{1}$ waves. Although there is a significant overall relative uncertainty between the $\pi^{-}$and $\pi^{0}$ production data, associated with luminosity and other systematic effects, the combined system is actually over determined [8]. As a consequence, if an acceptable solution is achieved it would support the approximations made in our analysis, such as the neglect of higher partial waves, $d-d$ interference, and the effect of coupling between the ${ }^{3} P_{2}$ and ${ }^{3} F_{2}$ partial waves.

The best fit to the combined $p p \rightarrow\{p p\}_{S} \pi^{0}$ and $p n \rightarrow\{p p\}_{s} \pi^{-}$data sets is obtained with

$$
\begin{aligned}
& M_{s}^{P}=(55.3 \pm 0.4)-(14.7 \pm 0.1) i \sqrt{\mathrm{nb} / \mathrm{sr}}, \\
& M_{d}^{P}=-(26.6 \pm 1.1)-(8.6 \pm 0.4) i \sqrt{\mathrm{nb} / \mathrm{sr}}, \\
& M_{d}^{F}=5.3 \pm 2.3 \sqrt{\mathrm{nb} / \mathrm{sr}}, \\
& M_{p}^{S}=-(32.4 \pm 2.2)+(17.3 \pm 2.7) i \sqrt{\mathrm{nb} / \mathrm{sr}}, \\
& M_{p}^{D}=-(109.6 \pm 9.6)+(140.7 \pm 4.0) i \sqrt{\mathrm{nb} / \mathrm{sr}} .
\end{aligned}
$$

Since this solution has $\chi^{2} / \mathrm{NDF}=89 / 82$, it shows that our truncated expansions can give a very good description of the data. The contribution from the ${ }^{3} F_{2} \rightarrow{ }^{1} S_{0} d$ transition is clearly very small and, if one eliminated $M_{d}^{F}$ completely, it would give only a marginally poorer fit with $\chi^{2} / \mathrm{NDF}=94 / 82$. It should be noted that, whereas the phases of $M_{s}^{P}, M_{d}^{P}$ and $M_{d}^{F}$ were imposed (see above), the phases of $M_{p}^{S}$ and $M_{p}^{D}$ were extracted from the production data.

\section{Measurement of $A_{x, x}$ and $A_{y, y}$ in $\vec{n} \vec{p} \rightarrow\{p p\}_{s} \pi^{-}$}

The spin-correlation coefficients $A_{x, x}$ and $A_{y, y}$ of the quasi-free $\vec{n} \vec{p} \rightarrow\{p p\}_{s} \pi^{-}$reaction were measured at ANKE in 2011 by using the vector polarised deuteron beam and the hydrogen Polarised Internal Target (PIT) [12], equipped with a long storage cell. The main source of background is the interaction of a beam particle with the aluminum storage cell walls. In order to obtain the shape of the background in the missing mass spectra, a dedicated measurement was made with the $\mathrm{N}_{2}$ gas in the storage cell.

As shown in Ref. [5], the experiment is essentially self-analysing. The values of $A_{x, x}$ and $A_{y, y}$ depend on the product of the beam and target polarisations. However, this product can be controlled by demanding that $A_{y, y}=1$ for all angles and $A_{x, x}=1$ in the forward and backward directions. This property of the experiment gives us also a powerful tool to study the systematic uncertainties of the measurement. Nevertheless, the beam and target polarisation values can be determined independently through the simultaneous detection of processes for which the analysing powers are known. For the beam energy of interest, $T_{n}=353 \mathrm{MeV}$ per nucleon, the most suitable reaction for the ANKE conditions is quasi-free $p n \rightarrow d \pi^{0}$.

The observed experimental asymmetry can be written as:

$$
\xi=\frac{\Sigma_{1}-\Sigma_{2}}{\Sigma_{2}-\Sigma_{2}}, \quad \xi / P Q=A_{x, x} \sin ^{2} \varphi+A_{y, y} \cos ^{2} \varphi
$$

where $\Sigma_{1}=N_{\uparrow \uparrow}+N_{\downarrow \downarrow}$ and $\Sigma_{2}=N_{\uparrow \downarrow}+N_{\downarrow \uparrow}$, with $N$ being the number of events collected with the directions of the beam and target spins indicated by the arrows. $P Q$ is the product of the beam and target polarisations. The value of $P Q=0.35$, extracted from the polarimetry reaction data, was used in the preliminary analysis.

The data were divided into five bins in the pion emission angle and $\xi / P Q$ was fitted as a function of $\cos ^{2} \varphi$ in each bin. The acceptance of the apparatus was significantly higher for events with large $\cos ^{2} \varphi$ so that the value of $A_{y, y}$ could be determined with smaller uncertainty than that of $A_{x, x}$. The $A_{y, y}$ results are shown in Fig. 5 in terms of of the pion emission angle. As expected, $A_{y, y}$ is consistent with unity over the whole angular range. To reduce the uncertainty in the extraction of $A_{x, x}$, it was assumed that $A_{y, y}=1$ and the $\cos ^{2} \varphi$ fit repeated, leading to the results shown in Fig. 6 .

For the $n p \rightarrow\{p p\}_{s} \pi^{-}$process, $\left(1-A_{x, x}\right) \cdot d \sigma / d \Omega \sim|\delta|^{2} \sin ^{2} \vartheta_{\pi}$, where $|\delta|^{2}$ is the square of one of the $p$-wave production amplitudes that is linked to the $4 N \pi$ contact interaction [5]. The differential cross 


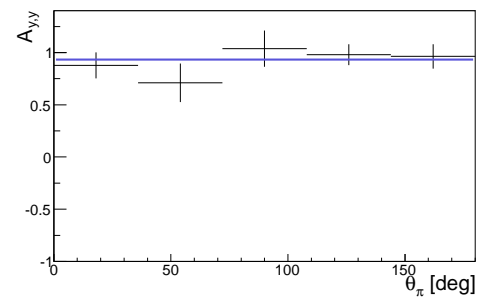

Fig. 5. The spin-correlation coefficient $A_{y, y}$ for the $\vec{n} \vec{p} \rightarrow\{p p\}_{s} \pi^{-}$reaction measured at $353 \mathrm{MeV}$ (preliminary).

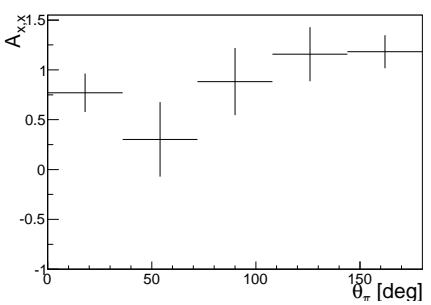

Fig. 6. The spin-correlation coefficient $A_{x, x}$ for the $\vec{n} \vec{p} \rightarrow\{p p\}_{s} \pi^{-}$reaction measured at $353 \mathrm{MeV}$ (preliminary).

section and the preliminary spin-correlation data give $\left(1-A_{x, x}\right) \cdot d \sigma / d \Omega\left(90^{\circ}\right)=(77 \pm 25) \mathrm{nb} / \mathrm{sr}$. This is fully consistent with the results of the partial wave analysis [8], which predicts a value of $52 \mathrm{nb} / \mathrm{sr}$.

\section{Outlook}

The theoretical uncertainties inherent in the assumptions made in the partial wave analysis [8] are hard to estimate. These can be tested by studying additional observables but the only non-trivial unmeasured one is the mixed spin-correlation coefficient $A_{z, x}$. Its measurement in the $\vec{p} \vec{n} \rightarrow\{p p\}_{s} \pi^{-}$ reaction is proposed as the next step in the ANKE pion production programme [13]. This result will provide new constrains on the partial wave analysis and therefore make the analysis more robust. The experiment will require a longitudinally polarised proton beam at the ANKE target position and this can be accomplished with the use of a Siberian snake. The installation of such a snake is foreseen for late 2012 .

The results presented here would not have been possible without the efforts of the COSY machine crew and other members of the ANKE collaboration. The work was partially supported by the COSYFFE programme and by grants from the DFG (436 RUS 113/965/0-1) and GNSF (ST09-1024-4-200).

\section{References}

1. S. Barsov et al., Nucl. Instrum. Methods Phys. Res. A 462 (2001) 364.

2. S. Dymov, COSY proposal \#192, (2008). This and other ANKE proposals are available from wWw.fz-juelich.de/ikp/anke/en/proposals.shtml.

3. A. Kulikov, COSY proposal \#158 (2008).

4. C. Hanhart, Phys. Rep. 397 (2004) 155.

5. S. Dymov, COSY proposal \#205 (2010).

6. D. Tsirkov et al., Phys. Lett. B 712 (2012) 370.

7. R. Bilger et al., Nucl. Phys. A 693 (2001) 633.

8. S. Dymov et al., Phys. Lett. B 712 (2012) 375.

9. F. Duncan et al., Phys. Rev. Lett. 80 (1998) 4390.

10. H. Hahn et al., Phys. Rev. C 53 (1996) 1074.

11. H. Hahn et al., Phys. Rev. Lett. 82 (1999) 2258.

12. R. Engels et al., AIP Conf. Proc. 1149 (2009) 890.

13. S. Dymov and A. Kacharava, COSY proposal \#213 (2012). 